\title{
OPEN Soil bacterial community composition in rice-fish integrated farming systems with different planting years
}

\author{
Zheng Zhao, Changbin Chu, Deping Zhou, Qingfeng Wang, Shuhang Wu ${ }^{凶}$, Xianqing Zheng, \\ Ke Song \& Weiguang Lv
}

The high productivity and efficient nutrient utilization in rice-fish integrated farming system are well reported. However, the characteristics of soil bacterial communities and their relationship with soil nutrient availability in rice-fish field remain unclear. In this study, we selected three paddy fields, including a rice monoculture field and two rice-fish fields with different planting years, to investigate the soil bacterial community composition with Illumina MiSeq sequencing technology. The results indicated that the soil properties were significantly different among different rice farming systems. The soil bacterial community composition in the rice-fish field was significantly different from that in the rice monoculture field. Five of the top 15 phyla were observed with significant differences and Nitrospirae was the most significant one. However, no taxa observed with significance between the rice planting area and aquaculture area no matter in the 1st or 5 th year of rice-fish field. RDA analysis showed that the soil bacterial community differentiation in the 5 th year of rice-fish field was positively correlated with soil properties, such as AN and OM contents, EC and pH value. Although the rice yields in rice-fish field decreased, the net economic benefit of the rice-fish system enhanced obviously due to the high value of aquaculture animals.

The rice-fish integrated farming system is an ancient agricultural production pattern and has been practiced for more than 2000 years in China ${ }^{1,2}$. In 2005, this system was recognized as a globally important agricultural heritage system (GIAHS) ${ }^{3}$. Recently, the use of rice-fish integrated farming has been gradually rising due to its exceptionally benefits, including positive effects on weed and pest control, reduced agrochemical inputs, improved soil nutrient availability, and increased yields and economic benefits ${ }^{4-6}$. The area of rice-fish fields in China is $1.67 \times 10^{6}$ ha, which accounts for approximately $4.5 \%$ of the total rice planting area in China ${ }^{7}$. Many studies have demonstrated that rice-fish integrated farming is an economical and eco-friendly agricultural pattern alternative to rice monoculture ${ }^{8,9}$.

Microorganisms play crucial roles in the formation of soil structure, decomposition of soil organic matter, circulation of soil nutrients, and crop health and growth ${ }^{10}$. The soil microbial community composition can reflect the quality of the soil ecological environment and directly affect soil fertility ${ }^{11}$. However, most previous studies related to rice-fish integrated farming systems have focused only on productivity or environmental benefits ${ }^{12,13}$ rather than complex soil organisms such as soil microbial communities. To date, there are few reports available about the characterization of the soil bacterial diversity and composition in rice-fish integrated farming systems. Therefore, it is necessary to study the community characteristics of soil microorganisms in rice-fish system, as well as their relationship with soil properties and productivity.

Chongming Eco-island, the third largest island in China, is located in the northeast of Shanghai city. Rice-fish integrated farming was introduced to the island in 2005, and yellow finless eel (Monopterus albus) and loach (Misgurnus spp.) are the main fish species cultured in paddy fields. In this study, the evolutionary characteristics of the soil bacterial communities in the rice-fish integrated farming systems were investigated using Illumina MiSeq sequencing. Our hypothesis is the soil bacterial community composition in rice-fish integrated farming system was greatly changed compared with rice monoculture. These differentiations of soil bacterial may relate to some soil factors or crucial taxa, which may further influence the productivity of the system. To test 


\begin{tabular}{|l|l|c|c|l|l|}
\hline Treatments & $\mathbf{A N}(\mathbf{m g} / \mathbf{k g})$ & $\mathbf{A P}(\mathbf{m g} / \mathbf{k g})$ & $\mathbf{A K}(\mathbf{m g} / \mathbf{k g})$ & $\mathbf{O M}(\%)$ & $\mathbf{p H}$ \\
\hline RM & $193.67 \pm 12.12 \mathrm{c}$ & $13.44 \pm 0.84 \mathrm{a}$ & $70.60 \pm 3.72 \mathrm{~b}$ & $1.21 \pm 0.15 \mathrm{ab}$ & $7.99 \pm 0.10 \mathrm{a}$ \\
\hline OP & $197.65 \pm 8.48 \mathrm{c}$ & $5.95 \pm 1.44 \mathrm{~b}$ & $150.40 \pm 9.56 \mathrm{a}$ & $1.02 \pm 0.10 \mathrm{ab}$ & $7.27 \pm 0.18 \mathrm{~b}$ \\
\hline OA & $209.59 \pm 9.55 \mathrm{bc}$ & $6.28 \pm 1.66 \mathrm{~b}$ & $146.40 \pm 16.44 \mathrm{a}$ & $0.98 \pm 0.12 \mathrm{~b}$ & $7.25 \pm 0.10 \mathrm{~b}$ \\
\hline FP & $246.73 \pm 18.51 \mathrm{a}$ & $4.77 \pm 1.11 \mathrm{~b}$ & $29.60 \pm 1.62 \mathrm{c}$ & $1.24 \pm 0.11 \mathrm{a}$ & $8.00 \pm 0.05 \mathrm{a}$ \\
\hline FA & $225.51 \pm 10.04 \mathrm{ab}$ & $5.59 \pm 0.94 \mathrm{~b}$ & $23.40 \pm 3.93 \mathrm{c}$ & $1.24 \pm 0.07 \mathrm{a}$ & $7.83 \pm 0.16 \mathrm{a}$ \\
\hline
\end{tabular}

Table 1. Soil properties in different rice systems and areas. Different letters within columns indicate significant differences at $P<0.05$.

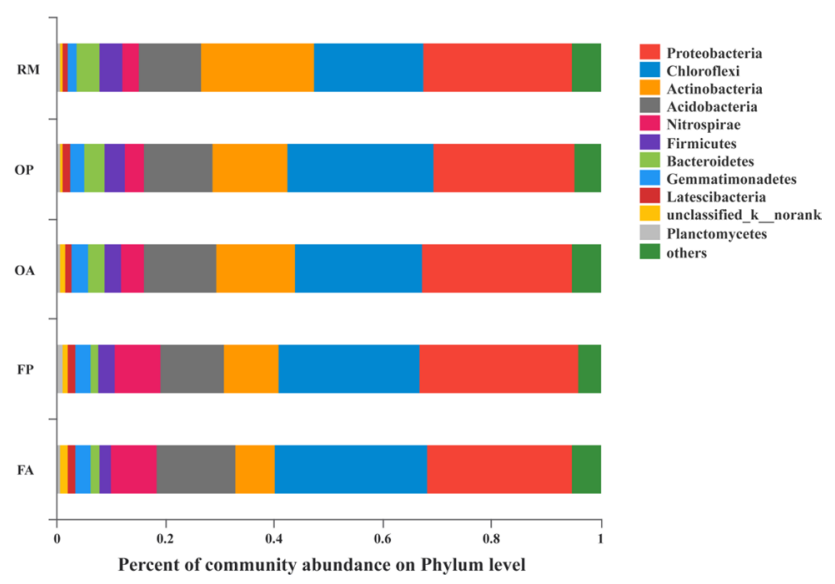

Figure 1. The average relative abundances on phylum level of soil bacterial communities in different rice systems and areas.

this hypothesis, a rice monoculture field and 2 rice-fish fields with different planting years ( 1 year and 5 years, respectively) on Chongming Eco-island were choose to investigate the soil bacterial community composition.

\section{Results}

Soil properties in different rice farming systems. Five treatments were designed in the three selected rice fields, including (1) rice monoculture field (RM); (2) planting area in the 1st year of rice-fish field (OP); (3) aquaculture area in the 1st year of rice-fish field (OA); (4) planting area in the 5th year of rice-fish field (FP); (5) aquaculture area in the 5th year of rice-fish field (FA). The soil properties of the five treatments were shown in Table 1. The highest soil available nitrogen (AN) content was observed in FP and was significantly higher than that in RM, OP and OA. The highest soil available phosphorus (AP) content was observed in RM and was significantly higher than that in the other 4 treatments. The highest soil available potassium (AK) content was measured in the 1st year of rice-fish field (OP and OA), followed by RM and the 5th year of rice-fish field (FP and FA), and significant differences were observed among different rice fields. The highest soil organic matter $(\mathrm{OM})$ content appeared in the 5th year of rice-fish field (FP and FA), and was only significantly higher than that in OA. In addition, the soil $\mathrm{pH}$ in the 1st year of rice-fish field (OP and $\mathrm{OA}$ ) was significantly lower than that in $\mathrm{RM}$ and the 5th year of rice-fish field (FP and FA). In summary, significant differences of soil properties were observed among the different rice farming systems.

Soil bacterial community composition. A total of $1,346,468$ sequences were obtained by $16 \mathrm{~S}$ rRNA MiSeq sequencing analysis after basal quality control (reads containing ambiguous bases were discarded; only overlapping sequences longer than $10 \mathrm{bp}$ were assembled; Operational taxonomic units (OTUs) were clustered with $97 \%$ similarity). These sequences were classified as 46 phyla, 800 genera and 5335 OTUs. As shown in Fig. 1, the dominant bacterial phyla across different treatments were Proteobacteria (26.06-29.41\%) and Chloroflexi (20.07-27.99\%), followed by Actinobacteria (7.22-20.87\%), Acidobacteria (11.36-14.46\%) and Nitrospirae (3.11-8.50\%). Since the implementation of rice-fish farming regime, the soil bacterial community composition has greatly changed. For example, Actinobacteria abundance decreased from $20.87 \%$ in RM to $7.22 \%$ in FA, while Nitrospirae abundance greatly increased from $3.11 \%$ in RM to $8.50 \%$ in FA. Between different areas in a same rice-fish field (i.e. OP vs OA or FP vs FA), the bacterial community composition were similar. The PCoA analysis on OTU level also showed that different areas within the same rice-fish field had high similarity in bacterial community composition. In contrast, the bacterial community composition differed distinctly among different rice farming systems (Fig. 2). Bacterial alpha diversity indices, as evaluated by Shannon, Simpson, ACE and Chao1, were shown in Table 2. Student's t-test was adopted to evaluate the difference among treatments. The 


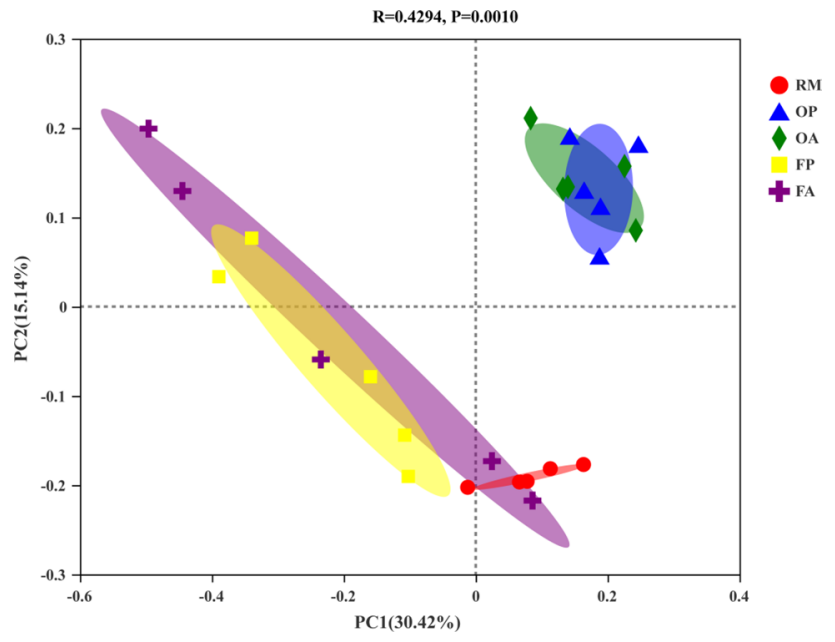

Figure 2. PCoA analysis on OTU level based on bray_curtis distance algorithm (significance among treatments were conducted with ANOSIM test, $\mathrm{R}=0.4294, P=0.0010$ ).

\begin{tabular}{|l|l|l|l|l|}
\hline Treatments & Shannon & Simpson & ACE & Chao1 \\
\hline RM & $6.73 \mathrm{ab}$ & $0.0066 \mathrm{a}$ & $3451.68 \mathrm{ab}$ & $3476.27 \mathrm{ab}$ \\
\hline OP & $6.97 \mathrm{a}$ & $0.0021 \mathrm{a}$ & $3450.48 \mathrm{ab}$ & $3488.81 \mathrm{ab}$ \\
\hline OA & $6.93 \mathrm{a}$ & $0.0023 \mathrm{a}$ & $3444.52 \mathrm{a}$ & $3486.72 \mathrm{a}$ \\
\hline FP & $6.59 \mathrm{~b}$ & $0.0044 \mathrm{a}$ & $3115.12 \mathrm{~b}$ & $3153.36 \mathrm{~b}$ \\
\hline FA & $6.60 \mathrm{ab}$ & $0.0049 \mathrm{a}$ & $3100.57 \mathrm{ab}$ & $3098.53 \mathrm{ab}$ \\
\hline
\end{tabular}

Table 2. Alpha diversity indices of soil bacterial in different rice systems and areas. Different letters within columns indicate significant differences at $P<0.05$.

results showed that the alpha indices of FP were significantly lower than other treatments, except for Simpson index.

Based on the Kruskal-Wallis test, the statistical differences among treatments were evaluated in the abundances of the top 15 phyla. The results showed that 5 phyla, including Actinobacteria, Nitrospirae, Bacteroidetes, Unclassified_k_norank and SBR1093 were observed significant differences among treatments, and the most significant phylum was Nitrospirae (Fig. 3). In order to trace the source of the significant differences, the Wilcoxon tests were conducted between every two rice cultivation patterns separately (Fig. 4). The results indicated that the significant differences were mainly derived from the comparison between RM and F_group (FP \& FA), as well as the comparison between the $\mathrm{O}_{\text {_group }}\left(\mathrm{OP} \& \mathrm{OA}\right.$ ) and $\mathrm{F}$ _group. In the comparison between the RM and $\mathrm{O}_{-}$ group, only the phylum Gemmatimonadetes was observed to have a significant difference. Furthermore, we also compared the differences of the top 15 phyla between planting area (P_group) and aquaculture area (A_group) within rice-fish fields, and the results showed no phyla observed with significant differences in the abundances.

Cluster analysis on genus level. The community heatmap of the top 30 genera is shown in Fig. 5. The genera Nitrospira, Anaerolineaceae and Acidobacteria showed higher abundances than the other genera. The community composition on genus level also differed markedly across the different experimental groups. The clustering tree indicates that the different areas in a same rice-fish field (i.e. OP vs OA or FP vs FA) showed high similarity on genera composition and clustered together first. Among the different rice farming system, the genera composition was clear distinct with each other. Moreover, the statistical difference among the 5 experimental groups of the top 30 genera was checked with Kruskal-Wallis test. The results showed that 11 genera were observed significant differences among treatments (Fig. 6, only significance phyla presented). Some genera, such as Nitrospira, norank_f_Nitrosomonadaceae, norank_c_Ardenticatenia and norank_o_NB1-j were enriched in the 5 years of rice-fish field (FP and FA), while some genera, such as Pseudarthrobacter, Sphingomonas and Nocardioides were enriched in RM. This results indicated that the soil bacterial community composition on genus level has changed greatly since the implementation of rice-fish farming regime, which is consist with previous analysis on phylum level. In addition, we used LEfSe analysis to show the differences in the taxa from the phylum to the genus level among the 5 experimental groups (Figure S1). A total of 150 taxa were observed to have significant differences in abundances, of which 60 taxa were enriched in RM, 27 taxa were enriched in OA, 24 taxa were enriched in FA, 22 taxa were enriched in OP and 17 taxa were enriched in FP. 


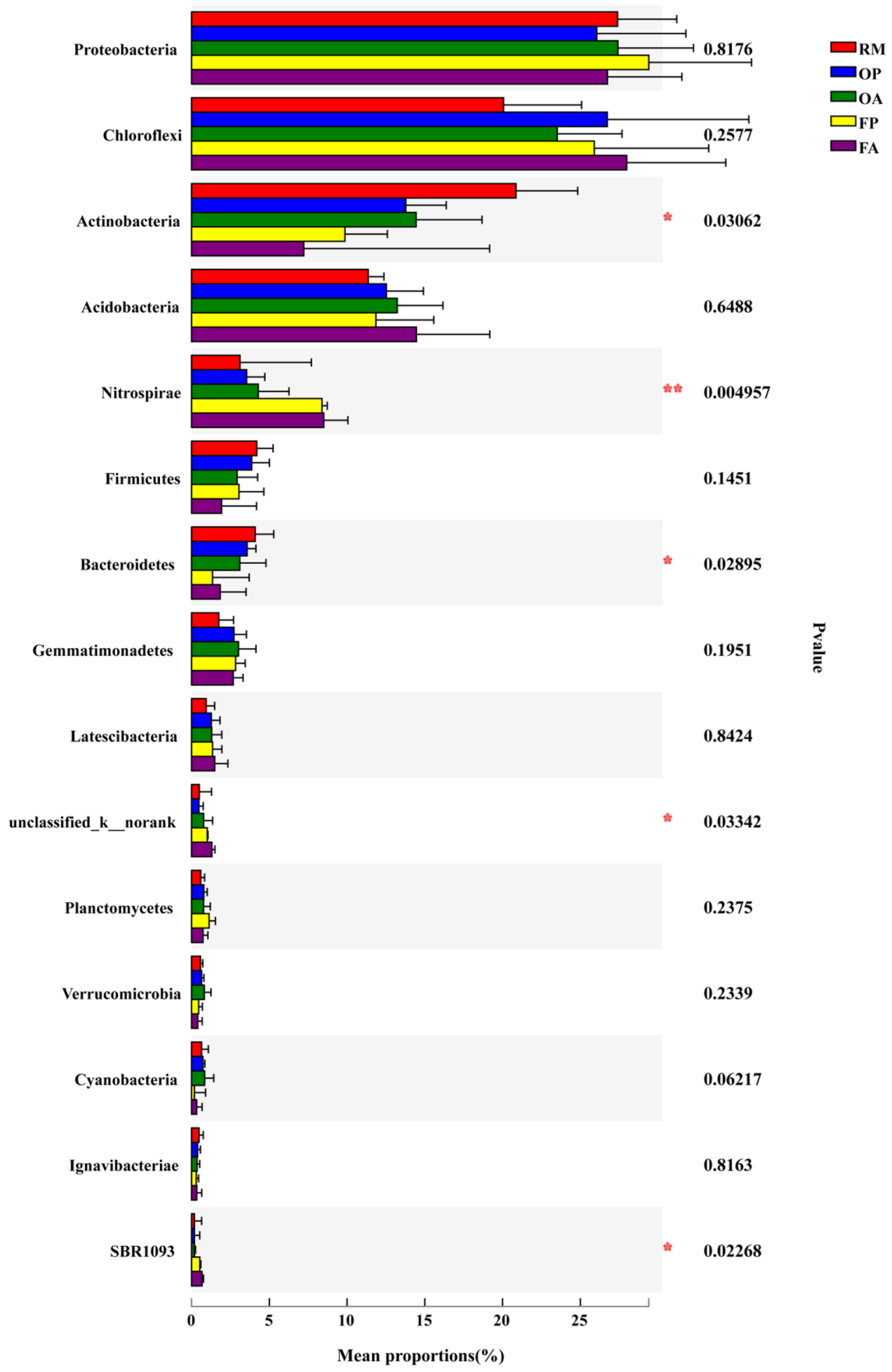

Figure 3. The differences with significance of the top 15 phyla in different rice systems and areas $\left({ }^{*}\right.$ indicates $0.01<P \leq 0.05,{ }^{* *}$ indicates $\left.0.001<P \leq 0.01\right)$.

Correlation between bacterial community composition and soil properties. Redundancy analysis (RDA) at the OTU level was performed to establish the linkages of soil properties with bacterial community composition (Fig. 7). The results showed that the soil properties together explained $32.99 \%$ of the total variations in bacterial community composition. The bacterial community in F_group (FP and FA) was positively correlated with soil factors, including $\mathrm{AN}$ and $\mathrm{OM}$ content, $\mathrm{EC}$ and $\mathrm{pH}$ value. In contrast, the bacterial community in O_group (OP and OA) was only positively correlated with soil AK content. In addition, the Mantel test was 

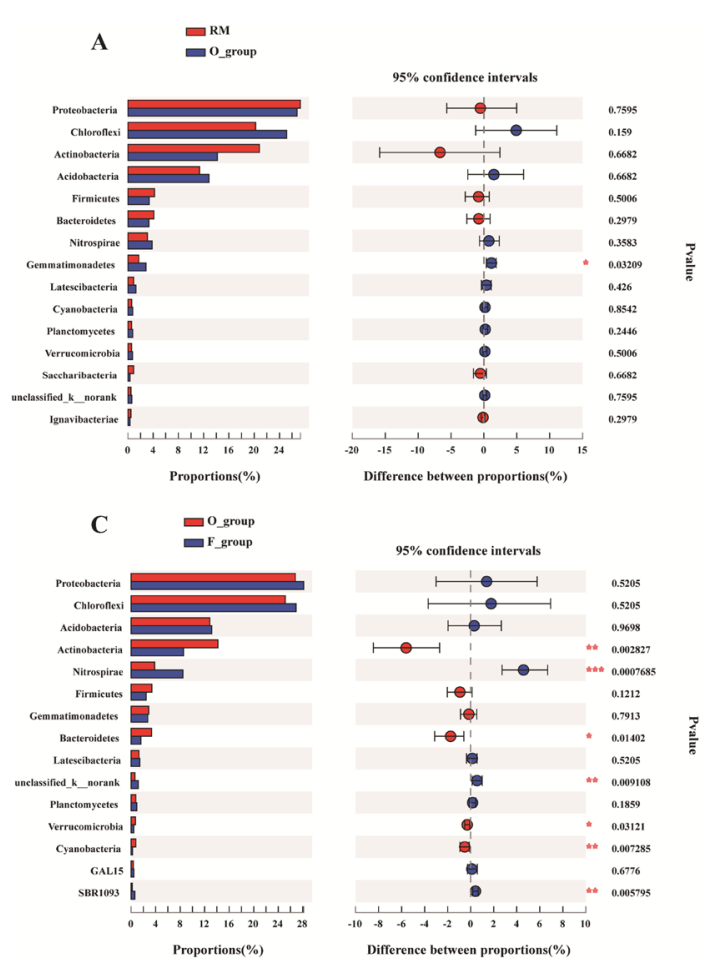
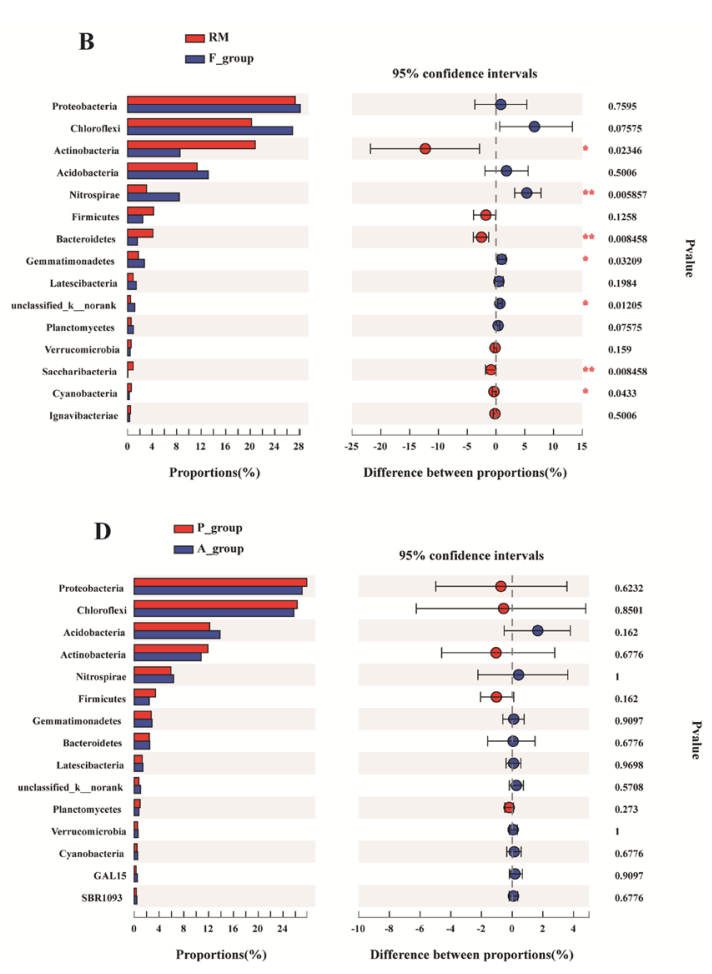

Figure 4. The differences with significance of the top 15 phyla between pairs of experimental groups (A: RM vs O_group, B: RM vs F_group, C: O_group vs F_group, D: P_group vs A_group; ${ }^{*}$ indicates $0.01<P \leq 0.05$, ** indicates $0.001<P \leq 0.01$, $\left.{ }^{* *} P \leq 0.001\right)$.

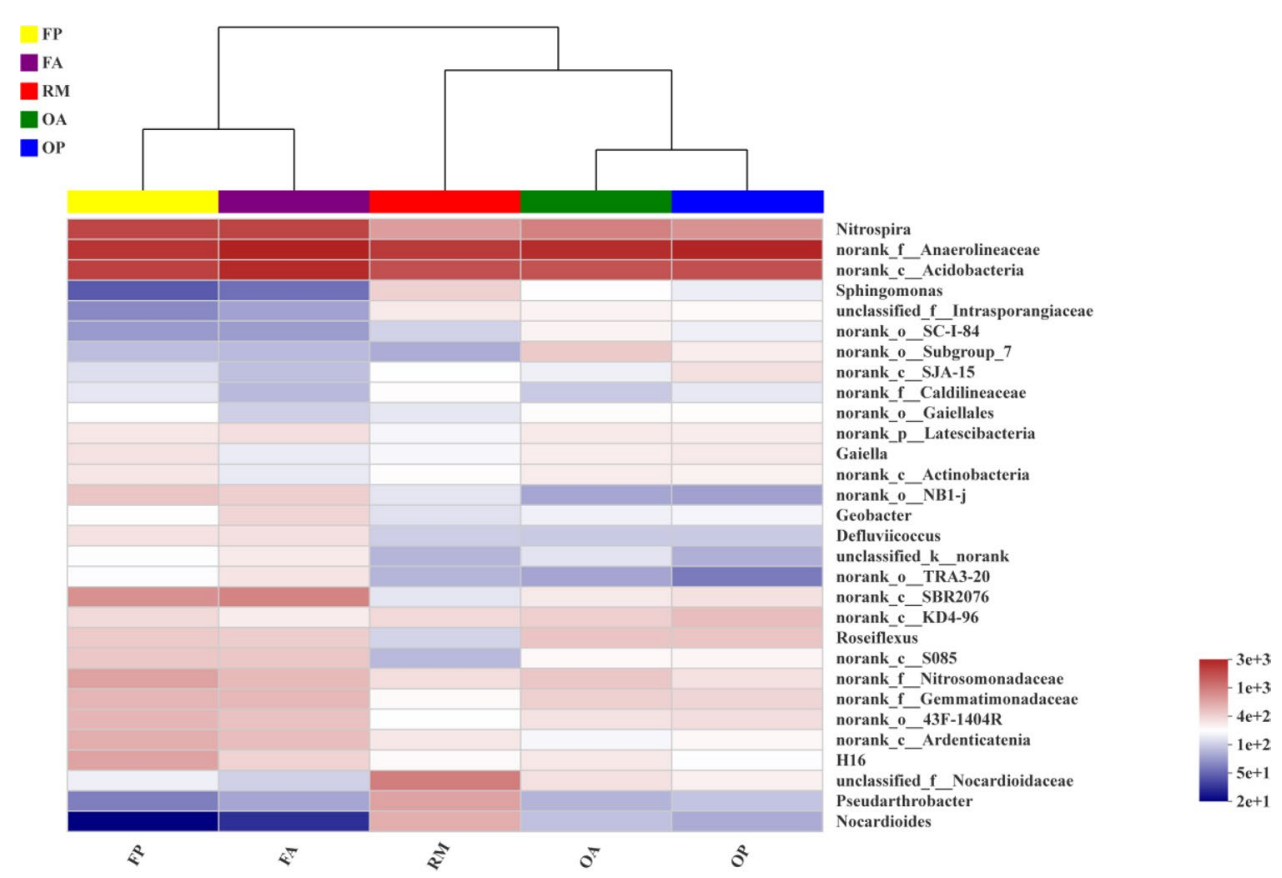

Figure 5. Heatmap of cluster analysis of the top 30 genera in different rice systems and areas.

employed to confirm the significance between soil factors and bacterial community composition. The results (Table 3$)$ indicated that the soil community composition was significantly $(P<0.05)$ correlated with the selected soil factors, except for soil AP content. Soil AK content was the most influential factor that correlated with bacterial community composition. 


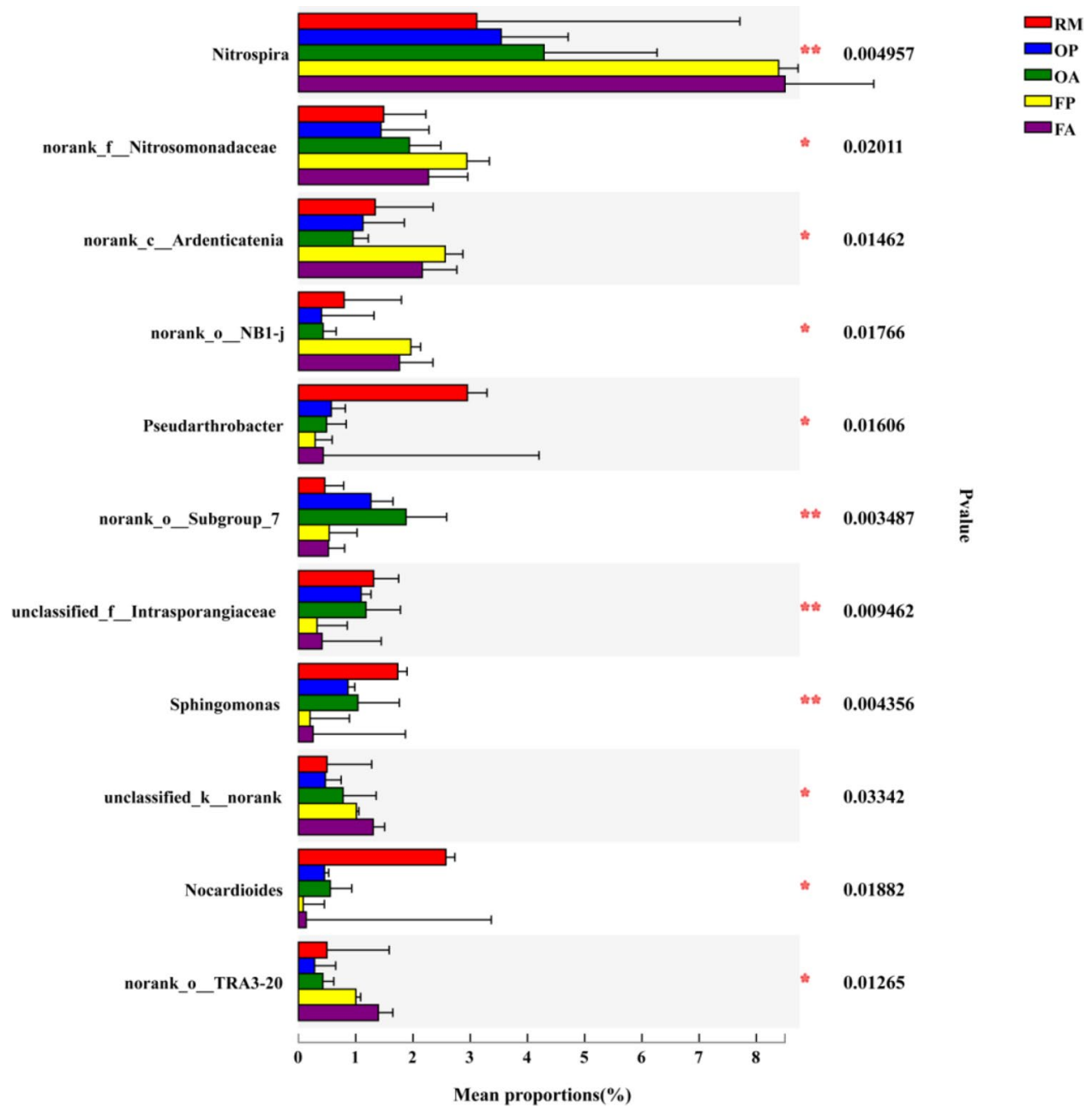

Figure 6. The differences with significance of the top 30 genera in different rice systems and areas ${ }^{*}$ indicates $0.01<P \leq 0.05,{ }^{* *}$ indicates $\left.0.001<P \leq 0.01\right)$.

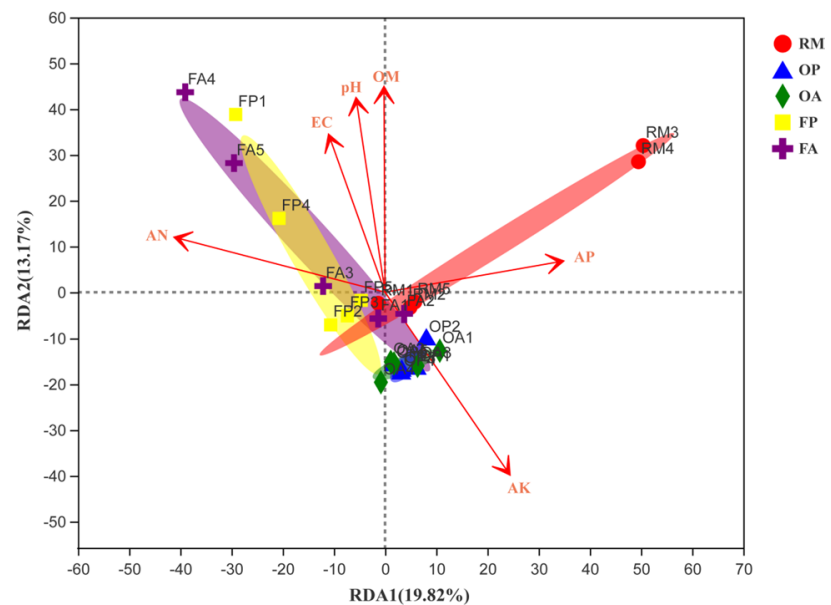

Figure 7. RDA analysis at the OTU level between the soil bacterial communities and soil properties. 


\begin{tabular}{|l|l|l|}
\hline Soil factors & r statistic & $\boldsymbol{P}$ value \\
\hline AN & 0.2872 & 0.014 \\
\hline AP & 0.1052 & 0.276 \\
\hline AK & 0.5510 & 0.001 \\
\hline OM & 0.2379 & 0.020 \\
\hline pH & 0.3142 & 0.002 \\
\hline EC & 0.1964 & 0.024 \\
\hline
\end{tabular}

Table 3. The correlations ( $r)$ and significance ( $P$ value) determined by Mantel test between the bacterial community composition and soil factors.

\begin{tabular}{|l|l|l|l|}
\hline Indicators & Rice monoculture & Rice-fish field in the 1st year & Rice-fish field in the 5th year \\
\hline Rice yield $\left(\mathrm{t} \cdot \mathrm{ha}^{-1}\right)$ & $7.99 \pm 0.23$ & $6.91 \pm 0.03$ & $7.45 \pm 0.29$ \\
\hline Protein content (\%) & $6.86 \pm 0.04$ & $7.07 \pm 0.07$ & $6.99 \pm 0.02$ \\
\hline Amylose content (\%) & $8.50 \pm 0.01$ & $8.30 \pm 0.06$ & $8.34 \pm 0.04$ \\
\hline Milled rice ratio (\%) & $69.30 \pm 0.27$ & $75.90 \pm 0.25$ & $78.17 \pm 0.22$ \\
\hline $\begin{array}{l}\text { Net economic benefit } \\
\left(1000 \mathrm{RMB} \cdot \mathrm{ha}^{-1} \cdot \mathrm{year}^{-1}\right)\end{array}$ & $20.51 \pm 0.71$ & $18.10 \pm 0.10$ & $23.23 \pm 0.45$ \\
\hline
\end{tabular}

Table 4. The rice yield, quality indices and net economic benefit of different rice farming systems.

Rice yield, quality and economic benefit. Rice yield, several quality indicators and the net economic benefit for the different rice cultivation regimes were also evaluated. As shown in Table 4, rice yield was decreased in rice-fish integrated farming systems, especially in the 1st year of rice-fish field. However, the net economic benefit in rice-fish field of the 5th year was increased due to the high economic value of aquatic animals. In the 1st year of rice-fish farming regime, the aquatic animal was not captured for sale as it had not yet reached the marketable size. Therefore, the net benefit of the 1st year of rice-fish field was lower than that in the rice monoculture. For the quality indicators, the protein content and milled rice ratio of rice-fish field were higher than rice monoculture, while the amylose content was opposite. More details for the quality and benefit analysis of rice-fish integrated farming system could be found in previous publication ${ }^{5}$.

\section{Discussion}

Developing Chongming Island into a world-class ecological island is very important for the urban development of Shanghai city. However, one challenge that needs to be overcome to achieve this goal is the high rate of agrochemical applications during the conventional agriculture production on the island. The rice-fish integrated farming regime could address this challenge by providing a means to diversify agricultural and aquacultural production, with increased yields and economic benefits mainly achieved by increased nutrient recycling and decreased agrochemical input ${ }^{5,14}$. The rice-fish fields generally exhibited improved productivity and enhanced ecological services, which have been reported in many previous studies ${ }^{15,16}$. However, the fundamental mechanisms for these enhancements in the rice-fish fields are not well-studied, which may limit the further promotion of rice-fish integrated farming regimes.

Soil microorganisms play an important role in regulating soil fertility by changing the diversity and structural composition of soil microbial communities ${ }^{11,17}$. Our study results with Illumina MiSeq sequencing indicated that soil bacterial community composition in rice-fish field was significantly different with rice monoculture, especially after long-term implementation of rice-fish regime (i.e. 5 years). Five phyla in the top 15 phyla and 11 genera in the top 30 genera were observed with significant differences among treatments. From phylum to the genus level, 150 taxa in total were detected to have significant differences in the abundances. This result indicated that the structure of the soil bacterial community was greatly changed after the rice-fish integrated farming regime was adopted in paddy field. Soil factors, such as AN content may play a crucial role in the differentiation of soil bacterial community composition, which has been supported by RDA analysis. Many researches have demonstrated that the interaction between functional bacteria and soil nutrients supply could enhance the productivity of agricultural systems ${ }^{18,19}$. However, this study was only provided the basic characteristics of soil bacterial community composition in rice-fish field. The bacterial functions and its relation with soil nutrients supply in the rice-fish field were not studied in depth. The phylum Nitrospirae, which observed with the most significance among different rice farming systems, need further studies to explore its functions in soil $\mathrm{N}$ transformation in rice-fish field. Previous research has shown that functional bacteria can decompose soil mineral $\mathrm{N}$ and improve nutrient availability, thereby promoting nutrient absorption by crops ${ }^{18}$.

Another interesting result in this study is no significant differences of the soil bacterial community composition were observed between the planting area and aquaculture area in rice-fish fields. This finding means that the differentiation of the soil bacterial communities in the rice-fish fields occurred throughout the whole system, not solely in the aquaculture area. This could be attributed to the deeper water and continuous flooding (i.e., no aeration period) in the rice-fish fields, which connected the whole system and provided an interactive 


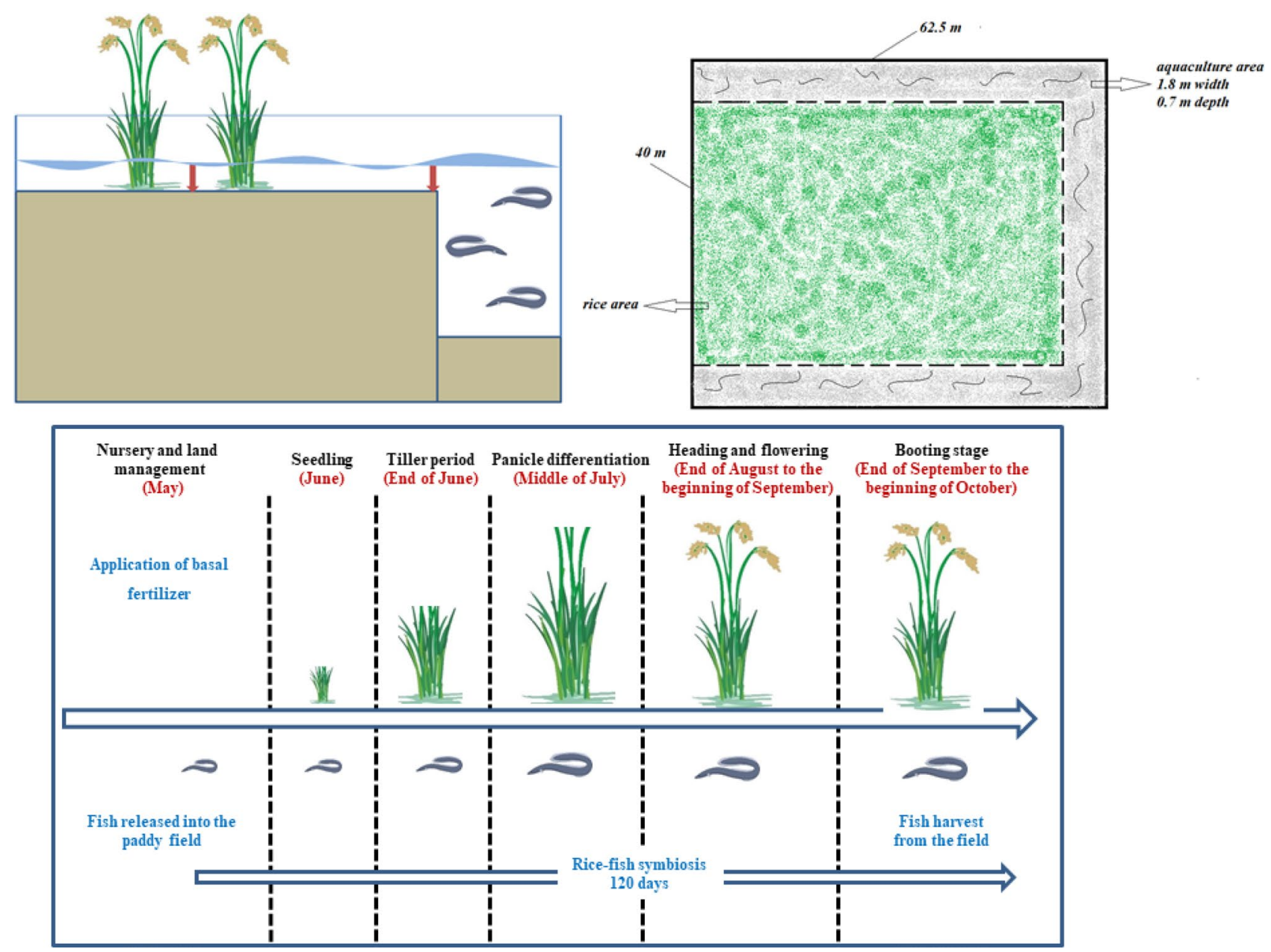

Figure 8. Layout and sequence diagram of the rice-fish integrated farming system (the red arrow indicates the sampling site).

environment for the various chemical and biological processes in the soil. This may be another key factor in the high productivity of rice-fish integrated farming system.

\section{Conclusion}

As the implementation of rice-fish integrated farming regime in Chongming Eco-island, the soil properties and bacterial community composition in rice-fish field was significantly different with that in rice monoculture. Significant differences of soil bacterial communities were also observed at both phylum level and genus level among different treatments. Soil properties, such as AN contents, play an important role in the differentiation of soil bacterial composition in rice-fish field. However, this study is only a preliminary exploration on the basic characteristics of soil bacterial community composition in rice-fish integrated farming system and its relationship with soil properties. Further studies are still needed in the direct linkage between soil nutrients supply and the crucial functional microorganisms, thus to reveal the mechanisms of high productivity of the rice-fish integrated system.

\section{Methods}

Experimental field description. The experimental site is located in Sanxing town, Chongming Ecoisland, Shanghai, China $\left(31^{\circ} 46^{\prime} 52^{\prime \prime} \mathrm{N}, 121^{\circ} 15^{\prime} 17^{\prime \prime} \mathrm{E}\right)$. This area has a subtropical humid monsoon climate, with a daily average air temperature of $15.6^{\circ} \mathrm{C}$ and annual precipitation of $1008 \mathrm{~mm}$. The soil type in this region is classified as Anthrosol based on Chinese Soil Taxonomy. Paddy rice is one of the typical food crops on the island, and the rice cultivation pattern is changing from monoculture to rice-fish integrated farming in recent years. Three paddy fields, including a rice monoculture field and two rice-fish fields with different planting years (1 year and 5 years), were selected to investigate soil bacterial community characteristics. The layout (Fig. 8 ) of the rice-fish field $(40.0 \mathrm{~m} \times 62.5 \mathrm{~m})$ consisted of a rice planting area $(38.2 \mathrm{~m} \times 58.9 \mathrm{~m})$ and surrounded on three sides by an aquaculture ditch $(0.7 \mathrm{~m}$ in depth and $1.8 \mathrm{~m}$ in width). The ratio of the rice area to the aquaculture area was 9:1. Yellow finless eel and loach were cultured in the aquaculture area during the rice growing season. Including 2 $\mathrm{kg}$ of deep yellow finless eel fry and $2 \mathrm{~kg}$ of loach fry were released into the aquaculture area that surrounding the rice fields. In the first year of rice-fish farming system, the finless eel and loach were not captured as they had not 
reached marketable size. Then, fish will captured from late September to early October before the rice harvest in every rice season (except the first season). The management sequence diagram of the rice-fish regime was shown in Fig. 8. Rice-fallow rotation was performed in the experimental paddy fields following the local conventional agricultural practices. The rice variety of "Qingxiangruangeng" is cultivated in these selected paddy fields. During rice season, each paddy field received $390 \mathrm{~kg} \mathrm{ha}^{-1}$ of compound fertilizer $(15 \% \mathrm{~N}, 15 \% \mathrm{P}$ and $15 \% \mathrm{~K})$ as the basal fertilizer, and $75 \mathrm{~kg} \mathrm{ha}^{-1}$ of urea $(46 \% \mathrm{~N})$ was used as topdressing at the seedling, tillering, elongation and booting stages. Flooding irrigation was adopted during the rice season. The rice monoculture field was managed with a midseason aeration period. During this period, the rice field is drained for approximately 10 days, and then re-flooding until the rice is ripe. In contrast, no midseason aeration was conducted in the two rice-fish fields, which carried out continuous flooding for aquaculture.

Soil sampling and measurements. In August of the 2018 rice season, soil samples were collected from the top-layer $(0-20 \mathrm{~cm})$ of the 5 designed treatments, including (1) RM: the rice monoculture field; (2) OP: the planting area in the 1st year of rice-fish field; ((3) OA: the aquaculture area in the 1st year of rice-fish field; (4) FP: the planting area in the 5th year of rice-fish field; (5) FA: the aquaculture area in the 5th year of rice-fish field. The soil sampling sites are indicated in the schematic diagram of the rice-fish integrated farming system (Fig. 8). Five duplicates were collected for each treatment, and 25 soil samples were collected in total. The soil samples were brought back to laboratory immediately in a cold storage box for the extraction of soil microbial DNA. In addition, soil properties, including organic matter $(\mathrm{OM})$, soil $\mathrm{pH}$, available nitrogen (AN), available phosphorus (AP) and available potassium (AK) contents, were also measured with standard experimental methods. The correlation between the soil bacterial communities and soil available nutrients was also analyzed with RDA analysis.

Soil DNA extraction, PCR amplification and sequencing. Soil microbial DNA was extracted using an E.Z.N.A. ${ }^{\circledR}$ soil DNA Kit (Omega Biotek, Norcross, GA, U.S.). The final DNA concentration and purification were determined by a NanoDrop 2000 UV-Vis spectrophotometer (Thermo Scientific, Wilmington, USA), and DNA quality was checked by $1 \%$ agarose gel electrophoresis ${ }^{20}$. The V3-V4 hypervariable regions of the bacterial 16S rRNA gene were amplified with primers 338F (5'-ACTCCTACGGGAGGCAGCAG-3') and 806R (5'GGACTACHVGGGTWTCTAAT-3') by a thermocycler PCR system (GeneAmp 9700, ABI, USA) ${ }^{21}$. The PCRs were conducted as follows: 3 min of denaturation at $95^{\circ} \mathrm{C}, 27$ cycles of $30 \mathrm{~s}$ at $95^{\circ} \mathrm{C}, 30 \mathrm{~s}$ for annealing at 55 ${ }^{\circ} \mathrm{C}$, and $45 \mathrm{~s}$ for elongation at $72{ }^{\circ} \mathrm{C}$, and a final extension at $72{ }^{\circ} \mathrm{C}$ for $10 \mathrm{~min}^{22}$. The resulting PCR products were extracted from a $2 \%$ agarose gel and further purified using an AxyPrep DNA Gel Extraction Kit (Axygen Biosciences, Union City, CA, USA). The purified amplicons were pooled in equimolar amounts and paired-end sequenced $(2 \times 300)$ on an Illumina MiSeq platform (Illumina, San Diego,USA) according to the standard protocols described by Majorbio Bio-Pharm Technology Co., Ltd. (Shanghai, China). The raw reads were deposited into the NCBI Sequence Read Archive (SRA) database (accession number: PRJNA635212).

Data processing and analysis. The raw 16S rRNA gene sequencing reads were demultiplexed, qualityfiltered by fastp version $0.20 .0^{23}$ and merged by FLASH version $1.2 .7^{24}$ with the following criteria: (i) the $300 \mathrm{bp}$ reads were truncated at any site receiving an average quality score of $<20$ over a $50 \mathrm{bp}$ sliding window, and the truncated reads shorter than 50 bp were discarded, reads containing ambiguous characters were also discarded; (ii) only overlapping sequences longer than $10 \mathrm{bp}$ were assembled according to their overlapped sequence. The maximum mismatch ratio of overlap region is 0.2 . Reads that could not be assembled were discarded; (iii) Samples were distinguished according to the barcode and primers, and the sequence direction was adjusted, exact barcode matching, 2 nucleotide mismatch in primer matching. Operational taxonomic units (OTUs) with 97\% similarity cutoff ${ }^{25}, 26$ were clustered using UPARSE version $7.1^{25}$, and chimeric sequences were identified and removed. The taxonomy of each OTU representative sequence was analyzed by RDP Classifier version $2.2^{27}$ against the $16 \mathrm{~S}$ rRNA database (Silva v138) using confidence threshold of $70 \%$. In addition, ANOSIM test, Kruskal-Wallis test, Wilcoxon tests, Student's t-test and Mantel test were employed to quantify the statistical differences among treatments.

Received: 12 January 2021; Accepted: 10 May 2021

Published online: 25 May 2021

\section{References}

1. Kangmin, L. Rice-fish culture in China: A review. Aquaculture 71, 173-186. https://doi.org/10.1016/0044-8486(88)90257-8 (1988).

2. Xie, J. et al. Conservation of traditional rice varieties in a globally important agricultural heritage system (GIAHS): Rice-fish coculture. Agricultural Sciences in China 10, 754-761. https://doi.org/10.1016/S1671-2927(11)60059-X (2011).

3. Ren, W. et al. Preservation of the genetic diversity of a local common carp in the agricultural heritage rice-fish system. Proc. Natl. Acad. Sci. USA 115, 546-554 (2018).

4. Hu, L. et al. Can the co-cultivation of rice and fish help sustain rice production?. Sci. Rep. 6, 28728 (2016).

5. Wan, N.-F. et al. Ecological intensification of rice production through rice-fish co-culture. J. Clean. Prod. 234, 1002-1012. https:// doi.org/10.1016/j.jclepro.2019.06.238 (2019).

6. Feng, J., Li, F., Zhou, X., Xu, C. \& Fang, F. Nutrient removal ability and economical benefit of a rice-fish co-culture system in aquaculture pond. Ecol. Eng. 94, 315-319. https://doi.org/10.1016/j.ecoleng.2016.06.002 (2016).

7. Liangliang, H. et al. Development of rice-fish system: Today and tomorrow. Chin. J. Eco-Agric. 23, 268-275. https://doi.org/10. 13930/j.cnki.cjea.150025 (2015). 
8. Hu, L. et al. Productivity and the complementary use of nitrogen in the coupled rice-crab system. Agric. Syst. 178, 102742. https:// doi.org/10.1016/j.agsy.2019.102742 (2020).

9. Bashir, M. A. et al. Co-culture of rice and aquatic animals: An integrated system to achieve production and environmental sustainability. J. Clean. Prod. 249, 119310. https://doi.org/10.1016/j.jclepro.2019.119310 (2020).

10. Chen, Y. et al. Distribution of bacterial communities across plateau freshwater lake and upslope soils. J. Environ. Sci. 43, 61-69. https://doi.org/10.1016/j.jes.2015.08.012 (2016).

11. Wang, R. et al. Bacterial community structure and functional potential of rhizosphere soils as influenced by nitrogen addition and bacterial wilt disease under continuous sesame cropping. Appl. Soil. Ecol. 125, 117-127. https://doi.org/10.1016/j.apsoil.2017.12. 014 (2018).

12. Hu, L. et al. The productivity of traditional rice-fish co-culture can be increased without increasing nitrogen loss to the environment. Agr. Ecosyst. Environ. 177, 28-34. https://doi.org/10.1016/j.agee.2013.05.023 (2013).

13. Zhang, J. et al. Rice-soft shell turtle coculture effects on yield and its environment. Agr. Ecosyst. Environ. 224, 116-122. https://doi. org/10.1016/j.agee.2016.03.045 (2016)

14. Berg, H. \& Tam, N. T. Decreased use of pesticides for increased yields of rice and fish-options for sustainable food production in the Mekong Delta. Sci. Total Environ. 619-620, 319-327. https://doi.org/10.1016/j.scitotenv.2017.11.062 (2018).

15. Liu, D. et al. Valuation of ecosystem services of rice-fish coculture systems in Ruyuan County, China. Ecosyst. Serv. 41, 101054. https://doi.org/10.1016/j.ecoser.2019.101054 (2020).

16. Li, F. et al. Impact of rice-fish/shrimp co-culture on the $\mathrm{N} 2 \mathrm{O}$ emission and $\mathrm{NH} 3$ volatilization in intensive aquaculture ponds. Sci. Total Environ. 655, 284-291. https://doi.org/10.1016/j.scitotenv.2018.10.440 (2019).

17. Bowles, T. M., Acosta-Martínez, V., Calderón, F. \& Jackson, L. E. Soil enzyme activities, microbial communities, and carbon and nitrogen availability in organic agroecosystems across an intensively-managed agricultural landscape. Soil Biol. Biochem. 68, 252-262 (2014).

18. Zhang, D. et al. Linking plant ecological stoichiometry with soil nutrient and bacterial communities in apple orchards. Appl. Soil. Ecol. 126, 1-10. https://doi.org/10.1016/j.apsoil.2017.12.017 (2018).

19. Fu, X., Wang, J., Sainju, U. M., Zhao, F. \& Liu, W. Soil microbial community and carbon and nitrogen fractions responses to mulching under winter wheat. Appl. Soil. Ecol. 139, 64-68 (2019).

20. Yang, Y. X., Mu, C. L., Luo, Z. \& Zhu, W. Y. Bromochloromethane, a methane analogue, affects the gut microbiota and metabolic profiles of the rat gastrointestinal tract. Appl. Environ. Microbiol. 82, 778-787 (2015).

21. Singh, K. M. et al. Microbial profiles of liquid and solid fraction associated biomaterial in buffalo rumen fed green and dry roughage diets by tagged 16S rRNA gene pyrosequencing. Mol. Biol. Rep. 42, 95-103 (2015).

22. Huang, B., Long, J., Liao, H., Liu, L. \& Yang, R. Characteristics of bacterial community and function in paddy soil profile around antimony mine and its response to antimony and arsenic contamination. Int. J. Environ. Res. Public Health 16, 4883 (2019).

23. Chen, S., Zhou, Y., Chen, Y. \& Gu, J. fastp: An ultra-fast all-in-one FASTQ preprocessor. Bioinformatics 34, i884-i890 (2018).

24. Salzberg, S. L. FLASH: Fast length adjustment of short reads to improve genome assemblies. Bioinformatics 27, 2957-2963 (2011).

25. Edgar, R. C. UPARSE: Highly accurate OTU sequences from microbial amplicon reads. Nat. Methods 10, 996-998. https://doi.org/ 10.1038/nmeth.2604 (2013).

26. Stackebrandt, E. \& Goebel, B. M. Taxonomic note: A place for DNA-DNA reassociation and 16S rRNA sequence analysis in the present species definition in bacteriology. Int. J. Syst. Bacteriol 44, 846-849 (1994).

27. Wang, Q., Garrity, G. M., Tiedje, J. M. \& Cole, J. R. Naive Bayesian classifier for rapid assignment of rRNA sequences into the new bacterial taxonomy. Appl. Environ. Microbiol. 73, 5264-5267 (2007).

\section{Acknowledgements}

Financial support for this study was provided by the SAAS Program for the Excellent Research Team (SPERT2017A03), the National Natural Science Foundation of China (31901121) and the Shanghai Sailing program (19YF1442900). We also appreciate Majorbio Bio-Pharm Technology Co., Ltd. (Shanghai, China) for the sequencing work and data analysis.

\section{Author contributions}

Z.Z., S.W. and W.L. designed and conducted the experiments. C.C. and D.Z. contributed to the analyzing of the results. Z.Z. wrote the main manuscript text and prepared all figures. Q.W., X.Z. and K.S. contributed to the revising of the manuscript. All authors reviewed the manuscript.

\section{Competing interests}

The authors declare no competing interests.

\section{Additional information}

Supplementary Information The online version contains supplementary material available at https:/doi.org/ 10.1038/s41598-021-90370-9.

Correspondence and requests for materials should be addressed to S.W. or W.L.

Reprints and permissions information is available at www.nature.com/reprints.

Publisher's note Springer Nature remains neutral with regard to jurisdictional claims in published maps and institutional affiliations.

Open Access This article is licensed under a Creative Commons Attribution 4.0 International License, which permits use, sharing, adaptation, distribution and reproduction in any medium or
format, as long as you give appropriate credit to the original author(s) and the source, provide a link to the Creative Commons licence, and indicate if changes were made. The images or other third party material in this article are included in the article's Creative Commons licence, unless indicated otherwise in a credit line to the material. If material is not included in the article's Creative Commons licence and your intended use is not permitted by statutory regulation or exceeds the permitted use, you will need to obtain permission directly from the copyright holder. To view a copy of this licence, visit http://creativecommons.org/licenses/by/4.0/.

(C) The Author(s) 2021 